\title{
Synthesis and In Vitro Antiprotozoal Activity of Bisbenzofuran Cations.
}

Svetlana M. Bakunova, Stanislav A. Bakunov, Tanja Wenzler, Todd Barszcz, Karl A. Werbovetz, Reto Brun, James Edwin Hall, and Richard R. Tidwell

Department of Pathology and Laboratory Medicine, School of Medicine, The University of North Carolina, Chapel Hill, North Carolina 27599-7525, Division of Medicinal Chemistry and Pharmacognosy, College of Pharmacy, The Ohio State University, 500 West 12th Avenue, Columbus, Ohio 43210, USA, and Department of Medical Parasitology and Infection Biology, Swiss Tropical Institute, CH-4002 Basel, Switzerland

Tidwell@med.unc.edu

\section{Supporting Information}

\section{Table of Contents}

Pages S2 - S5: Elemental analysis data. 
Table 1. Elemental analysis data

\begin{tabular}{|c|c|c|c|c|c|c|c|}
\hline$\#$ & Formula & & $\% \mathrm{C}$ & $\% \mathrm{H}$ & $\% \mathbf{N}$ & $\% \mathrm{Cl}$ & other \\
\hline \multirow[t]{2}{*}{1} & \multirow{2}{*}{$\mathrm{C}_{19} \mathrm{H}_{16} \mathrm{~N}_{4} \mathrm{O}_{2} \cdot 2 \mathrm{HCl} \cdot 1 \mathrm{H}_{2} \mathrm{O}$} & calc & 53.91 & 4.76 & 13.24 & 16.75 & \\
\hline & & found & 53.98 & 4.46 & 13.14 & 16.68 & \\
\hline \multirow[t]{2}{*}{2} & \multirow[t]{2}{*}{$\mathrm{C}_{25} \mathrm{H}_{28} \mathrm{~N}_{4} \mathrm{O}_{2} \cdot 2 \mathrm{HCl} \cdot 1.5 \mathrm{H}_{2} \mathrm{O} \cdot 0.5 \mathrm{C}_{2} \mathrm{H}_{5} \mathrm{OH}$} & calc & 57.88 & 6.73 & 10.38 & 13.14 & \\
\hline & & found & 57.93 & 6.56 & 10.05 & 12.93 & \\
\hline \multirow[t]{2}{*}{3} & \multirow{2}{*}{$\mathrm{C}_{23} \mathrm{H}_{20} \mathrm{~N}_{4} \mathrm{O}_{2} \cdot 2 \mathrm{HCl} \cdot 0.5 \mathrm{H}_{2} \mathrm{O}$} & calc & 59.24 & 4.97 & 12.01 & 15.20 & \\
\hline & & found & 59.13 & 5.06 & 12.01 & 15.17 & \\
\hline \multirow[t]{2}{*}{4} & \multirow[t]{2}{*}{$\mathrm{C}_{19} \mathrm{H}_{16} \mathrm{~N}_{4} \mathrm{O}_{2} \cdot 2 \mathrm{HCl} \cdot 0.6 \mathrm{H}_{2} \mathrm{O}$} & calc & 54.85 & 4.64 & 13.46 & 17.04 & \\
\hline & & found & 54.62 & 4.75 & 13.21 & 17.17 & \\
\hline \multirow[t]{2}{*}{5} & \multirow{2}{*}{$\mathrm{C}_{25} \mathrm{H}_{28} \mathrm{~N}_{4} \mathrm{O}_{2} \cdot 2 \mathrm{HCl} \cdot 1.5 \mathrm{H}_{2} \mathrm{O}$} & calc & 58.14 & 6.44 & 10.85 & 13.73 & \\
\hline & & found & 58.26 & 6.47 & 10.79 & 13.88 & \\
\hline \multirow[t]{2}{*}{6} & \multirow{2}{*}{$\mathrm{C}_{23} \mathrm{H}_{20} \mathrm{~N}_{4} \mathrm{O}_{2} \cdot 2 \mathrm{HCl} \cdot 1.1 \mathrm{H}_{2} \mathrm{O}$} & calc & 57.89 & 5.11 & 11.74 & 14.86 & \\
\hline & & found & 57.69 & 5.24 & 11.78 & 15.01 & \\
\hline \multirow[t]{2}{*}{7} & \multirow{2}{*}{$\mathrm{C}_{23} \mathrm{H}_{20} \mathrm{~N}_{4} \mathrm{O}_{2} \cdot 2 \mathrm{HCl} \cdot 2 \mathrm{H}_{2} \mathrm{O}$} & calc & 55.99 & 5.31 & 11.36 & 14.37 & \\
\hline & & found & 56.16 & 5.23 & 11.39 & 14.22 & \\
\hline \multirow[t]{2}{*}{8} & \multirow{2}{*}{$\mathrm{C}_{20} \mathrm{H}_{18} \mathrm{~N}_{4} \mathrm{O}_{2} \cdot 2 \mathrm{HCl} \cdot 1.3 \mathrm{H}_{2} \mathrm{O}$} & calc & 54.26 & 5.15 & 12.65 & 16.02 & \\
\hline & & found & 54.22 & 5.13 & 12.50 & 16.25 & \\
\hline \multirow[t]{2}{*}{9} & \multirow{2}{*}{$\mathrm{C}_{26} \mathrm{H}_{30} \mathrm{~N}_{4} \mathrm{O}_{2} \cdot 2 \mathrm{HCl}$} & calc & 62.03 & 6.41 & 11.13 & 14.08 & \\
\hline & & found & 61.91 & 6.34 & 11.03 & 14.23 & \\
\hline \multirow[t]{2}{*}{10} & \multirow{2}{*}{$\mathrm{C}_{24} \mathrm{H}_{22} \mathrm{~N}_{4} \mathrm{O}_{2} \cdot 2 \mathrm{HCl} \cdot 1.7 \mathrm{H}_{2} \mathrm{O}$} & calc & 57.42 & 5.50 & 11.16 & 14.12 & \\
\hline & & found & 57.28 & 5.47 & 11.09 & 14.23 & \\
\hline \multirow[t]{2}{*}{11} & \multirow{2}{*}{$\mathrm{C}_{20} \mathrm{H}_{18} \mathrm{~N}_{4} \mathrm{O}_{2} \cdot 2 \mathrm{HCl} \cdot 2 \mathrm{H}_{2} \mathrm{O}$} & calc & 52.76 & 5.31 & 12.30 & 15.57 & \\
\hline & & found & 52.68 & 5.22 & 12.54 & 15.86 & \\
\hline \multirow[t]{2}{*}{12} & \multirow{2}{*}{$\mathrm{C}_{26} \mathrm{H}_{30} \mathrm{~N}_{4} \mathrm{O}_{2} \cdot 2 \mathrm{HCl} \cdot 2.5 \mathrm{H}_{2} \mathrm{O}$} & calc & 56.93 & 6.80 & 10.21 & 12.93 & \\
\hline & & found & 56.78 & 6.58 & 10.09 & 13.08 & \\
\hline 13 & $\mathrm{C}_{24} \mathrm{H}_{22} \mathrm{~N}_{4} \mathrm{O}_{2} \cdot 2 \mathrm{HCl} \cdot 2.5 \mathrm{H}_{2} \mathrm{O}$ & calc & 55.82 & 5.66 & 10.85 & 13.73 & \\
\hline & & found & 55.82 & 5.43 & 10.88 & 13.84 & \\
\hline 14 & $\mathrm{C}_{20} \mathrm{H}_{18} \mathrm{~N}_{4} \mathrm{O}_{2} \cdot 2 \mathrm{HCl} \cdot 1 \mathrm{H}_{2} \mathrm{O}$ & calc & 54.93 & 5.07 & 12.81 & 16.21 & \\
\hline & & found & 54.86 & 4.93 & 12.68 & 16.47 & \\
\hline 15 & $\mathrm{C}_{26} \mathrm{H}_{30} \mathrm{~N}_{4} \mathrm{O}_{2} \cdot 2 \mathrm{Cl} \cdot 1.2 \mathrm{H}_{2} \mathrm{O}$ & calc & 59.48 & 6.60 & 10.67 & 13.50 & \\
\hline & & found & 59.34 & 6.42 & 10.56 & 13.83 & \\
\hline 16 & $\mathrm{C}_{24} \mathrm{H}_{22} \mathrm{~N}_{4} \mathrm{O}_{2} \cdot 1.9 \mathrm{HCl} \cdot 1 \mathrm{H}_{2} \mathrm{O}$ & calc & 59.34 & 5.37 & 11.53 & 13.87 & \\
\hline & & found & 59.22 & 5.13 & 11.25 & 13.91 & \\
\hline 17 & $\mathrm{C}_{21} \mathrm{H}_{20} \mathrm{~N}_{4} \mathrm{O}_{2} \cdot 2 \mathrm{HCl} \cdot 1 \mathrm{H}_{2} \mathrm{O}$ & calc & 55.88 & 5.36 & 12.41 & 15.71 & \\
\hline & & found & 55.88 & 5.29 & 12.30 & 15.74 & \\
\hline 18 & $\mathrm{C}_{27} \mathrm{H}_{32} \mathrm{~N}_{4} \mathrm{O}_{2} \cdot 2 \mathrm{HCl} \cdot 1 \mathrm{H}_{2} \mathrm{O}$ & calc & 60.56 & 6.78 & 10.46 & 13.24 & \\
\hline & & found & 60.64 & 6.65 & 10.32 & 13.39 & \\
\hline 19 & $\mathrm{C}_{25} \mathrm{H}_{24} \mathrm{~N}_{4} \mathrm{O}_{2} \cdot 2 \mathrm{HCl} \cdot 1.2 \mathrm{H}_{2} \mathrm{O}$ & calc & 59.22 & 5.65 & 11.05 & 14.03 & \\
\hline & & found & 59.10 & 5.59 & 11.11 & 14.29 & \\
\hline 20 & $\mathrm{C}_{21} \mathrm{H}_{20} \mathrm{~N}_{4} \mathrm{O}_{2} \cdot 2 \mathrm{HCl} \cdot 0.3 \mathrm{H}_{2} \mathrm{O}$ & calc & 57.49 & 5.19 & 12.77 & 16.16 & \\
\hline & & found & 57.52 & 5.14 & 12.55 & 16.16 & \\
\hline 21 & $\mathrm{C}_{27} \mathrm{H}_{32} \mathrm{~N}_{4} \mathrm{O}_{2} \cdot 2 \mathrm{HCl} \cdot 1 \mathrm{H}_{2} \mathrm{O}$ & calc & 60.56 & 6.78 & 10.46 & 13.24 & \\
\hline & & found & 60.70 & 6.75 & 10.30 & 12.99 & \\
\hline
\end{tabular}




\begin{tabular}{|c|c|c|c|c|c|c|c|}
\hline \multirow[t]{2}{*}{22} & \multirow{2}{*}{$\mathrm{C}_{25} \mathrm{H}_{24} \mathrm{~N}_{4} \mathrm{O}_{2} \cdot 2 \mathrm{HCl} \cdot 2 \mathrm{H}_{2} \mathrm{O}$} & calc & 57.59 & 5.80 & 10.74 & 13.60 & \\
\hline & & found & 57.70 & 5.62 & 10.77 & 13.67 & \\
\hline \multirow[t]{2}{*}{23} & \multirow{2}{*}{$\mathrm{C}_{22} \mathrm{H}_{22} \mathrm{~N}_{4} \mathrm{O}_{2} \cdot 2 \mathrm{HCl} \cdot 0.5 \mathrm{H}_{2} \mathrm{O}$} & calc & 57.90 & 5.52 & 12.28 & 15.54 & \\
\hline & & found & 57.90 & 5.27 & 11.98 & 15.60 & \\
\hline \multirow[t]{2}{*}{24} & \multirow{2}{*}{$\mathrm{C}_{28} \mathrm{H}_{34} \mathrm{~N}_{4} \mathrm{O}_{2} \cdot 2 \mathrm{HCl} \cdot 1.2 \mathrm{H}_{2} \mathrm{O}$} & calc & 60.80 & 7.00 & 10.13 & 12.82 & \\
\hline & & found & 60.45 & 6.63 & 10.06 & 12.53 & \\
\hline \multirow[t]{2}{*}{25} & \multirow{2}{*}{$\mathrm{C}_{26} \mathrm{H}_{26} \mathrm{~N}_{4} \mathrm{O}_{2} \cdot 2 \mathrm{HCl} \cdot 2.5 \mathrm{H}_{2} \mathrm{O}$} & calc & 57.35 & 6.11 & 10.29 & 13.02 & \\
\hline & & found & 57.45 & 5.98 & 10.24 & 13.36 & \\
\hline \multirow[t]{2}{*}{26} & \multirow[t]{2}{*}{$\mathrm{C}_{22} \mathrm{H}_{22} \mathrm{~N}_{4} \mathrm{O}_{2} \cdot 2 \mathrm{HCl} \cdot 1.5 \mathrm{H}_{2} \mathrm{O}$} & calc & 55.70 & 5.74 & 11.81 & 14.95 & \\
\hline & & found & 55.50 & 5.56 & 11.71 & 15.42 & \\
\hline \multirow[t]{2}{*}{27} & \multirow[t]{2}{*}{$\mathrm{C}_{28} \mathrm{H}_{34} \mathrm{~N}_{4} \mathrm{O}_{2} \cdot 2 \mathrm{HCl} \cdot 0.5 \mathrm{H}_{2} \mathrm{O}$} & calc & 62.22 & 6.90 & 10.36 & 13.12 & \\
\hline & & found & 62.38 & 6.81 & 10.22 & 12.98 & \\
\hline \multirow[t]{2}{*}{28} & \multirow[t]{2}{*}{$\mathrm{C}_{26} \mathrm{H}_{26} \mathrm{~N}_{4} \mathrm{O}_{2} \cdot 2 \mathrm{HCl} \cdot 0.7 \mathrm{H}_{2} \mathrm{O}$} & calc & 60.99 & 5.79 & 10.94 & 13.85 & \\
\hline & & found & 60.96 & 5.72 & 10.77 & 13.71 & \\
\hline \multirow[t]{2}{*}{29} & \multirow[t]{2}{*}{$\mathrm{C}_{23} \mathrm{H}_{24} \mathrm{~N}_{4} \mathrm{O}_{2} \cdot 2 \mathrm{HCl} \cdot 1.6 \mathrm{H}_{2} \mathrm{O}$} & calc & 56.35 & 6.00 & 11.43 & 14.46 & \\
\hline & & found & 56.00 & 5.72 & 11.26 & 14.86 & \\
\hline \multirow[t]{2}{*}{30} & \multirow[t]{2}{*}{$\mathrm{C}_{29} \mathrm{H}_{36} \mathrm{~N}_{4} \mathrm{O}_{2} \cdot 2 \mathrm{HCl} \cdot 0.7 \mathrm{H}_{2} \mathrm{O}$} & calc & 62.40 & 7.12 & 10.04 & 12.70 & \\
\hline & & found & 62.34 & 7.05 & 9.96 & 12.93 & \\
\hline \multirow[t]{2}{*}{31} & \multirow{2}{*}{$\mathrm{C}_{27} \mathrm{H}_{28} \mathrm{~N}_{4} \mathrm{O}_{2} \cdot 2 \mathrm{HCl} \cdot 2 \mathrm{H}_{2} \mathrm{O}$} & calc & 59.02 & 6.24 & 10.20 & 12.90 & \\
\hline & & found & 58.79 & 6.29 & 10.09 & 12.75 & \\
\hline \multirow[t]{2}{*}{32} & \multirow{2}{*}{$\mathrm{C}_{25} \mathrm{H}_{28} \mathrm{~N}_{4} \mathrm{O}_{4} \cdot 2 \mathrm{HCl}$} & calc & 57.58 & 5.80 & 10.74 & 13.60 & \\
\hline & & found & 57.34 & 5.82 & 10.61 & 13.33 & \\
\hline \multirow[t]{2}{*}{33} & $\mathrm{C}_{31} \mathrm{H}_{40} \mathrm{~N}_{4} \mathrm{O}_{4} \cdot 2.1 \mathrm{HCl} \cdot 2.4 \mathrm{H}_{2} \mathrm{O}$ & calc & 57.06 & 7.25 & 8.59 & 11.41 & \\
\hline & & found & 56.96 & 7.00 & 8.50 & 11.48 & \\
\hline 34 & $\mathrm{C}_{29} \mathrm{H}_{32} \mathrm{~N}_{4} \mathrm{O}_{4} \cdot 2 \mathrm{HCl} \cdot 2.5 \mathrm{H}_{2} \mathrm{O}$ & calc & 56.31 & 6.36 & 9.06 & 11.46 & \\
\hline & & found & 56.05 & 6.14 & 9.03 & 11.34 & \\
\hline 35 & $\mathrm{C}_{23} \mathrm{H}_{24} \mathrm{~N}_{4} \mathrm{O}_{2} \cdot 2 \mathrm{HCl} \cdot 0.7 \mathrm{H}_{2} \mathrm{O}$ & calc & 58.28 & 5.83 & 11.82 & 14.96 & \\
\hline & & found & 58.40 & 6.15 & 11.60 & 14.75 & \\
\hline 36 & $\mathrm{C}_{29} \mathrm{H}_{36} \mathrm{~N}_{4} \mathrm{O}_{2} \cdot 2 \mathrm{HCl} \cdot 1.3 \mathrm{H}_{2} \mathrm{O}$ & calc & 61.22 & 7.19 & 9.85 & 12.46 & \\
\hline & & found & 61.33 & 7.24 & 9.64 & 12.24 & \\
\hline 37 & $\mathrm{C}_{27} \mathrm{H}_{28} \mathrm{~N}_{4} \mathrm{O}_{2} \cdot 2 \mathrm{HCl} \cdot 1.5 \mathrm{H}_{2} \mathrm{O} \cdot 0.3 i-\mathrm{PrOH}$ & calc & 60.00 & 6.39 & 10.03 & 12.70 & \\
\hline & & found & 60.07 & 6.25 & 10.05 & 12.40 & \\
\hline 38 & $\mathrm{C}_{24} \mathrm{H}_{26} \mathrm{~N}_{4} \mathrm{O}_{2} \cdot 2 \mathrm{HCl} \cdot 0.2 \mathrm{H}_{2} \mathrm{O}$ & calc & 60.18 & 5.98 & 11.70 & 14.80 & \\
\hline & & found & 59.92 & 6.02 & 11.66 & 15.03 & \\
\hline 39 & $\mathrm{C}_{25} \mathrm{H}_{28} \mathrm{~N}_{4} \mathrm{O}_{2} \cdot 2 \mathrm{HCl} \cdot 2 \mathrm{H}_{2} \mathrm{O}$ & calc & 57.14 & 6.52 & 10.66 & 13.49 & \\
\hline & & found & 56.91 & 6.51 & 10.52 & 13.64 & \\
\hline 40 & $\mathrm{C}_{26} \mathrm{H}_{30} \mathrm{~N}_{4} \mathrm{O}_{2} \cdot 2.3 \mathrm{HCl}$ & calc & 60.71 & 6.33 & 10.89 & 15.85 & \\
\hline & & found & 60.63 & 6.29 & 11.10 & 15.69 & \\
\hline 41 & $\mathrm{C}_{27} \mathrm{H}_{32} \mathrm{~N}_{4} \mathrm{O}_{2} \cdot 2 \mathrm{HCl} \cdot 1.5 \mathrm{H}_{2} \mathrm{O}$ & calc & 59.56 & 6.85 & 10.29 & 13.02 & \\
\hline & & found & 59.59 & 6.89 & 10.27 & 13.16 & \\
\hline 42 & $\mathrm{C}_{28} \mathrm{H}_{34} \mathrm{~N}_{4} \mathrm{O}_{2} \cdot 2 \mathrm{HCl} \cdot 0.2 \mathrm{H}_{2} \mathrm{O}$ & calc & 62.85 & 6.86 & 10.47 & 13.25 & \\
\hline & & found & 62.72 & 6.95 & 10.35 & 13.39 & \\
\hline 43 & $\mathrm{C}_{29} \mathrm{H}_{36} \mathrm{~N}_{4} \mathrm{O}_{2} \cdot 2.1 \mathrm{HCl} \cdot 1.3 \mathrm{H}_{2} \mathrm{O}$ & calc & 60.83 & 7.16 & 9.78 & 13.00 & \\
\hline & & found & 60.85 & 7.12 & 9.61 & 12.91 & \\
\hline 47 & $\mathrm{C}_{17} \mathrm{H}_{8} \mathrm{Br}_{2} \mathrm{O}_{3}$ & calc & 48.61 & 1.92 & & & $\begin{array}{l}\% \mathrm{Br} \\
38.04\end{array}$ \\
\hline
\end{tabular}




\begin{tabular}{|c|c|c|c|c|c|c|}
\hline & & found & 48.61 & 1.89 & & $\begin{array}{l}\% \mathrm{Br} \\
37.87\end{array}$ \\
\hline \multirow[t]{2}{*}{$\overline{48}$} & \multirow[t]{2}{*}{$\mathrm{C}_{17} \mathrm{H}_{8} \mathrm{Br}_{2} \mathrm{O}_{3}$} & calc & 48.61 & 1.92 & & $\begin{array}{l}\% \mathrm{Br} \\
38.04\end{array}$ \\
\hline & & found & 48.64 & 1.86 & & $\begin{array}{l}\% \mathrm{Br} \\
38.08\end{array}$ \\
\hline \multirow[t]{2}{*}{49} & \multirow[t]{2}{*}{$\mathrm{C}_{17} \mathrm{H}_{10} \mathrm{Br}_{2} \mathrm{O}_{3}$} & calc & 50.28 & 2.48 & & $\begin{array}{l}\% \mathrm{Br} \\
39.35\end{array}$ \\
\hline & & found & 50.44 & 2.42 & & $\begin{array}{l}\% \mathrm{Br} \\
39.23\end{array}$ \\
\hline \multirow[t]{2}{*}{$\overline{50}$} & \multirow[t]{2}{*}{$\mathrm{C}_{17} \mathrm{H}_{10} \mathrm{Br}_{2} \mathrm{O}_{3}$} & calc & 50.28 & 2.48 & & $\begin{array}{l}\% \mathrm{Br} \\
39.35\end{array}$ \\
\hline & & found & 50.42 & 2.58 & & $\begin{array}{l}\% \mathrm{Br} \\
39.4\end{array}$ \\
\hline \multirow[t]{2}{*}{51} & \multirow[t]{2}{*}{$\mathrm{C}_{19} \mathrm{H}_{10} \mathrm{~N}_{2} \mathrm{O}_{2}$} & calc & 76.50 & 3.38 & 9.39 & \\
\hline & & found & 76.39 & 3.35 & 9.33 & \\
\hline \multirow[t]{2}{*}{52} & \multirow{2}{*}{$\mathrm{C}_{19} \mathrm{H}_{10} \mathrm{~N}_{2} \mathrm{O}_{2} \cdot 0.3 \mathrm{H}_{2} \mathrm{O}$} & calc & 75.14 & 3.52 & 9.22 & \\
\hline & & found & 75.25 & 3.39 & 9.23 & \\
\hline \multirow[t]{2}{*}{56} & \multirow{2}{*}{$\mathrm{C}_{13} \mathrm{H}_{14} \mathrm{O}_{4} \cdot 0.1 \mathrm{H}_{2} \mathrm{O}$} & calc & 66.15 & 6.06 & & \\
\hline & & found & 66.15 & 5.91 & & \\
\hline \multirow[t]{2}{*}{57} & \multirow{2}{*}{$\mathrm{C}_{13} \mathrm{H}_{12} \mathrm{O}_{4} \cdot 0.1 \mathrm{H}_{2} \mathrm{O}$} & calc & 66.72 & 5.25 & & \\
\hline & & found & 66.50 & 5.11 & & \\
\hline \multirow[t]{2}{*}{58} & \multirow{2}{*}{$\mathrm{C}_{13} \mathrm{H}_{12} \mathrm{O}_{4}$} & calc & 67.21 & 5.21 & & \\
\hline & & found & 67.21 & 5.18 & & \\
\hline \multirow[t]{2}{*}{59} & \multirow[t]{2}{*}{$\mathrm{C}_{14} \mathrm{H}_{12} \mathrm{O}_{3} \cdot 0.2 \mathrm{H}_{2} \mathrm{O}$} & calc & 72.53 & 5.39 & & \\
\hline & & found & 72.50 & 5.26 & & \\
\hline \multirow[t]{2}{*}{60} & \multirow{2}{*}{$\mathrm{C}_{14} \mathrm{H}_{12} \mathrm{O}_{3} \cdot 0.1 \mathrm{H}_{2} \mathrm{O}$} & calc & 73.09 & 5.35 & & \\
\hline & & found & 72.97 & 5.33 & & \\
\hline \multirow[t]{2}{*}{61} & \multirow[t]{2}{*}{$\mathrm{C}_{22} \mathrm{H}_{18} \mathrm{O}_{6} \cdot 0.8 \mathrm{H}_{2} \mathrm{O}$} & calc & 67.27 & 5.03 & & \\
\hline & & found & 67.03 & 4.59 & & \\
\hline \multirow[t]{2}{*}{62} & \multirow[t]{2}{*}{$\mathrm{C}_{22} \mathrm{H}_{18} \mathrm{O}_{6}$} & calc & 69.83 & 4.79 & & \\
\hline & & found & 69.70 & 4.74 & & \\
\hline \multirow[t]{2}{*}{63} & \multirow[t]{2}{*}{$\mathrm{C}_{22} \mathrm{H}_{18} \mathrm{O}_{6}$} & calc & 69.83 & 4.79 & & \\
\hline & & found & 69.61 & 4.79 & & \\
\hline 64 & $\mathrm{C}_{20} \mathrm{H}_{12} \mathrm{~N}_{2} \mathrm{O}_{2} \cdot 0.6 \mathrm{H}_{2} \mathrm{O}$ & calc & 74.34 & 4.12 & 8.67 & \\
\hline & & found & 74.02 & 3.76 & 8.48 & \\
\hline 65 & $\mathrm{C}_{20} \mathrm{H}_{12} \mathrm{~N}_{2} \mathrm{O}_{2} \cdot 0.2 \mathrm{H}_{2} \mathrm{O}$ & calc & 76.04 & 3.96 & 8.87 & \\
\hline & & found & 75.96 & 3.76 & 8.83 & \\
\hline 66 & $\mathrm{C}_{20} \mathrm{H}_{12} \mathrm{~N}_{2} \mathrm{O}_{2} \cdot 0.1 \mathrm{H}_{2} \mathrm{O}$ & calc & 76.47 & 3.91 & 8.92 & \\
\hline & & found & 76.34 & 3.66 & 8.83 & \\
\hline 67 & $\mathrm{C}_{23} \mathrm{H}_{20} \mathrm{O}_{6} \cdot 0.5 \mathrm{H}_{2} \mathrm{O}$ & calc & 68.82 & 5.27 & & \\
\hline & & found & 68.94 & 5.13 & & \\
\hline 68 & $\mathrm{C}_{23} \mathrm{H}_{20} \mathrm{O}_{6}$ & calc & 70.40 & 5.14 & & \\
\hline & & found & 70.67 & 5.20 & & \\
\hline 69 & $\mathrm{C}_{24} \mathrm{H}_{22} \mathrm{O}_{6} \cdot 0.6 \mathrm{H}_{2} \mathrm{O}$ & calc & 69.09 & 5.60 & & \\
\hline & & found & 68.95 & 5.33 & & \\
\hline
\end{tabular}




\begin{tabular}{|c|c|c|c|c|c|c|c|}
\hline \multirow[t]{2}{*}{70} & \multirow[t]{2}{*}{$\mathrm{C}_{24} \mathrm{H}_{22} \mathrm{O}_{6}$} & calc & 70.92 & 5.46 & & & \\
\hline & & found & 70.70 & 5.49 & & & \\
\hline \multirow[t]{2}{*}{71} & \multirow[t]{2}{*}{$\mathrm{C}_{25} \mathrm{H}_{24} \mathrm{O}_{6}$} & calc & 71.41 & 5.75 & & & \\
\hline & & found & 71.33 & 5.85 & & & \\
\hline \multirow[t]{2}{*}{72} & \multirow[t]{2}{*}{$\mathrm{C}_{25} \mathrm{H}_{24} \mathrm{O}_{6}$} & calc & 71.41 & 5.75 & & & \\
\hline & & found & 71.10 & 5.76 & & & \\
\hline \multirow[t]{2}{*}{73} & \multirow{2}{*}{$\mathrm{C}_{21} \mathrm{H}_{14} \mathrm{~N}_{2} \mathrm{O}_{2} \cdot 0.2 \mathrm{EtOAc}$} & calc & 76.12 & 4.57 & 8.14 & & \\
\hline & & found & 75.75 & 4.29 & 8.27 & & \\
\hline \multirow[t]{2}{*}{74} & \multirow[t]{2}{*}{$\mathrm{C}_{21} \mathrm{H}_{14} \mathrm{~N}_{2} \mathrm{O}_{2}$} & calc & 77.29 & 4.32 & 8.58 & & \\
\hline & & found & 76.99 & 4.53 & 8.33 & & \\
\hline \multirow[t]{2}{*}{75} & \multirow[t]{2}{*}{$\mathrm{C}_{22} \mathrm{H}_{16} \mathrm{~N}_{2} \mathrm{O}_{2} \cdot 0.3 \mathrm{H}_{2} \mathrm{O}$} & calc & 76.42 & 4.84 & 8.10 & & \\
\hline & & found & 76.54 & 4.76 & 8.18 & & \\
\hline \multirow[t]{2}{*}{76} & \multirow[t]{2}{*}{$\mathrm{C}_{22} \mathrm{H}_{16} \mathrm{~N}_{2} \mathrm{O}_{2} \cdot 0.4 \mathrm{H}_{2} \mathrm{O}$} & calc & 76.02 & 4.87 & 8.06 & & \\
\hline & & found & 76.07 & 4.82 & 8.13 & & \\
\hline \multirow[t]{2}{*}{77} & \multirow[t]{2}{*}{$\mathrm{C}_{23} \mathrm{H}_{18} \mathrm{~N}_{2} \mathrm{O}_{2} \cdot 0.1 \mathrm{H}_{2} \mathrm{O}$} & calc & 77.55 & 5.15 & 7.86 & & \\
\hline & & found & 77.49 & 5.09 & 7.91 & & \\
\hline \multirow[t]{2}{*}{78} & \multirow[t]{2}{*}{$\mathrm{C}_{23} \mathrm{H}_{18} \mathrm{~N}_{2} \mathrm{O}_{2}$} & calc & 77.95 & 5.12 & 7.90 & & \\
\hline & & found & 78.24 & 5.44 & 7.58 & & \\
\hline \multirow[t]{2}{*}{80} & \multirow[t]{2}{*}{$\mathrm{C}_{25} \mathrm{H}_{24} \mathrm{O}_{6} \cdot 1 \mathrm{H}_{2} \mathrm{O}$} & calc & 68.48 & 5.98 & & & \\
\hline & & found & 68.51 & 5.71 & & & \\
\hline \multirow[t]{2}{*}{81} & \multirow{2}{*}{$\mathrm{C}_{25} \mathrm{H}_{26} \mathrm{~N}_{2} \mathrm{O}_{6} \cdot 0.3 \mathrm{H}_{2} \mathrm{O}$} & calc & 65.86 & 5.88 & 6.14 & & \\
\hline & & found & 65.86 & 5.70 & 5.98 & & \\
\hline \multirow[t]{2}{*}{82} & \multirow{2}{*}{$\mathrm{C}_{25} \mathrm{H}_{22} \mathrm{~N}_{2} \mathrm{O}_{4} \cdot 0.2 \mathrm{AcOH}$} & calc & 71.54 & 5.39 & 6.57 & & \\
\hline & & found & 71.53 & 5.45 & 6.13 & & \\
\hline 84 & $\mathrm{C}_{12} \mathrm{H}_{11} \mathrm{NO}_{2}$ & calc & 71.63 & 5.51 & 6.96 & & \\
\hline & & found & 71.68 & 5.49 & 7.00 & & \\
\hline 85 & $\mathrm{C}_{12} \mathrm{H}_{9} \mathrm{NO}_{2}$ & calc & 72.35 & 4.55 & 7.03 & & \\
\hline & & found & 72.14 & 4.55 & 6.97 & & \\
\hline 86 & $\mathrm{C}_{13} \mathrm{H}_{9} \mathrm{NO}$ & calc & 79.98 & 4.65 & 7.17 & & \\
\hline & & found & 79.88 & 4.62 & 7.20 & & \\
\hline 88 & $\mathrm{C}_{24} \mathrm{H}_{20} \mathrm{~N}_{2} \mathrm{O}_{2}$ & calc & 77.16 & 5.64 & 7.26 & & \\
\hline & & found & 77.19 & 5.73 & 6.92 & & \\
\hline 89 & $\mathrm{C}_{25} \mathrm{H}_{22} \mathrm{~N}_{2} \mathrm{O}_{2} \cdot 0.2 \mathrm{Hexane}$ & calc & 78.73 & 6.25 & 7.01 & & \\
\hline & & found & 78.88 & 6.06 & 6.85 & & \\
\hline 90 & $\mathrm{C}_{26} \mathrm{H}_{24} \mathrm{~N}_{2} \mathrm{O}_{2} \cdot 0.3 \mathrm{EtOAc}$ & calc & 77.25 & 6.29 & 6.62 & & \\
\hline & & found & 77.44 & 6.37 & 6.50 & & \\
\hline 91 & $\mathrm{C}_{27} \mathrm{H}_{26} \mathrm{~N}_{2} \mathrm{O}_{2}$ & calc & 79.00 & 6.38 & 6.82 & & \\
\hline & & found & 78.75 & 6.64 & 6.53 & & \\
\hline 92 & $\mathrm{C}_{28} \mathrm{H}_{28} \mathrm{~N}_{2} \mathrm{O}_{2} \cdot 0.1$ EtOAc & calc & 78.71 & 6.70 & 6.46 & & \\
\hline & & found & 78.74 & 7.06 & 6.17 & & \\
\hline 93 & $\mathrm{C}_{29} \mathrm{H}_{30} \mathrm{~N}_{2} \mathrm{O}_{2} \cdot 0.3 \mathrm{Hexane}$ & calc & 79.66 & 7.42 & 6.03 & & \\
\hline & & found & 79.60 & 7.17 & 5.82 & & \\
\hline
\end{tabular}

